\title{
Aggregation under a forced convective flow
}

\author{
Laura López-Tomàs, Josep Claret, Francesc Mas, and Francesc Sagués \\ Departament de Química-Física, Universitat de Barcelona, Martí i Franques 1, 08028 Barcelona, Spain
}

(Received 18 May 1992)

\begin{abstract}
Morphological transitions are analyzed for a radial multiparticle diffusion-limited aggregation process grown under a convective drift. The introduction of a tangential flow changes the morphology of the diffusion-limited structure, into multiarm structures, inclined opposite to the flow, whose limit consists of single arms, when decreasing density. The case of shear flow is also considered. The anisotropy of the patterns is characterized in terms of a tangential correlation function based analysis. Comparison between the simulation results and preliminary experimental results has been done.
\end{abstract}

\section{INTRODUCTION}

Since its introduction ten years ago, ${ }^{1}$ the diffusionlimited aggregation (DLA) model has been considered as a prototypical routine for simulating many nonequilibrium aggregation and deposition phenomena. ${ }^{2-10}$ However, the real complexity of many of these phenomena prompted, since the very beginning, successive generalizations of the original DLA code to take into account a variety of effects such as those related to particle drift, ${ }^{11,12}$ finite density of aggregation particles, ${ }^{13,14}$ sticking probability, ${ }^{15}$ or surface tension. ${ }^{16,17}$ In this paper we will present simulation and experimental results combining two of the previously mentioned effects: Multiparticle aggregation will be considered under a convective drift.

Being particularly interested in the electrodeposition phenomenon, our motivation traces back to the indications contained in the early papers by Brady and Ball ${ }^{18}$ and Sawada, Dougherty, and Gollub, ${ }^{19}$ where it was suggested that convection, in addition to the usual diffusion and migration effects, might play an important role on the morphology of the deposits. More recently, experiments with forced radial flow seem to provide some evidence of morphological transitions from the DLA fractal to the needle structure. ${ }^{20}$

In this paper we refer to a convection-diffusion-limited multiparticle aggregation process growing in radial geometry. The drift will be independently changed. The simulations are based on the use of random walkers, whose biased wandering results from the superimposed drift. The case of shear flow is also considered as a better candidate to explain experimental results here reported, obtained preliminarily with a rotating radial cell where two-dimensional $\mathrm{Zn}$ electrodeposition is conducted.

We consider that deposition takes place at a $2 R \times 2 R$ square lattice in which we define a centered circle of radius $R$. The deposit grows within this circle from the central seed point toward the outer boundary. At the beginning of the simulation, we fill at random the region defined by the circle with particles, until a density given by $C_{0}$ is reached. A bulk region is defined between a circle of radius $R$ and a circle of radius $(R-1)$ [see Fig. 1(a)]. At the end of each iteration, particles on this region are removed and this space is filled again at random until the prescribed $C_{0}$ density is attained. The motion of convective-diffusing particles can be described by random walks except for a bias resulting from the convection. Typically, on what follows, $R=256$ and the simulation proceeds in the standard way. When a particle is chosen, one of its neighbor sites is selected according to its probability as a possible next position. If unoccupied, the particle moves to this new site. If occupied, the particle remains fixed. If the new site borders the cluster, the particle becomes part of it. If the particle overpasses the bulk region, the particle is annihilated. The next iteration begins when all particles have been examined and the bulk restored. The process stops when the cluster reaches a radius of 150 units. The bias is incorporated through the displacement law

$$
\begin{aligned}
P(i, j)= & {\left[\frac{1-P_{x}-P_{y}}{4}\right] P(i+1, j) } \\
& +\left[P_{x}+\frac{1-P_{x}-P_{y}}{4}\right] P(i-1, j) \\
& +\left[\frac{1-P_{x}-P_{y}}{4}\right] P(i, j+1) \\
& +\left[P_{y}+\frac{1-P_{x}-P_{y}}{4}\right] P(i, j-1),
\end{aligned}
$$

where $P_{x}$ and $P_{y}$ are transformed from the original tangential and radial drifts by means of an appropriate trigonometric transformation. ${ }^{21}$

The simulations, restricted only to tangential flows, are shown in Figs. 2 and 3 and correspond to two different types of flow; in the first case, a constant flow is assumed for the overall region where particles move. In the second case, a more realistic treatment leads us to use a shear flow, which depends on the distance between the Brownian particle and the seed point.

In order to compare the behavior of a pure multiparticle DLA with the patterns obtained introducing these two types of flow, we also use a tangential correlation function based analysis following the standard definition

$$
C_{R}(\theta)=\frac{1}{N} \sum_{\theta^{\prime}} \rho_{R}\left(\theta+\theta^{\prime}\right) \rho_{R}\left(\theta^{\prime}\right),
$$



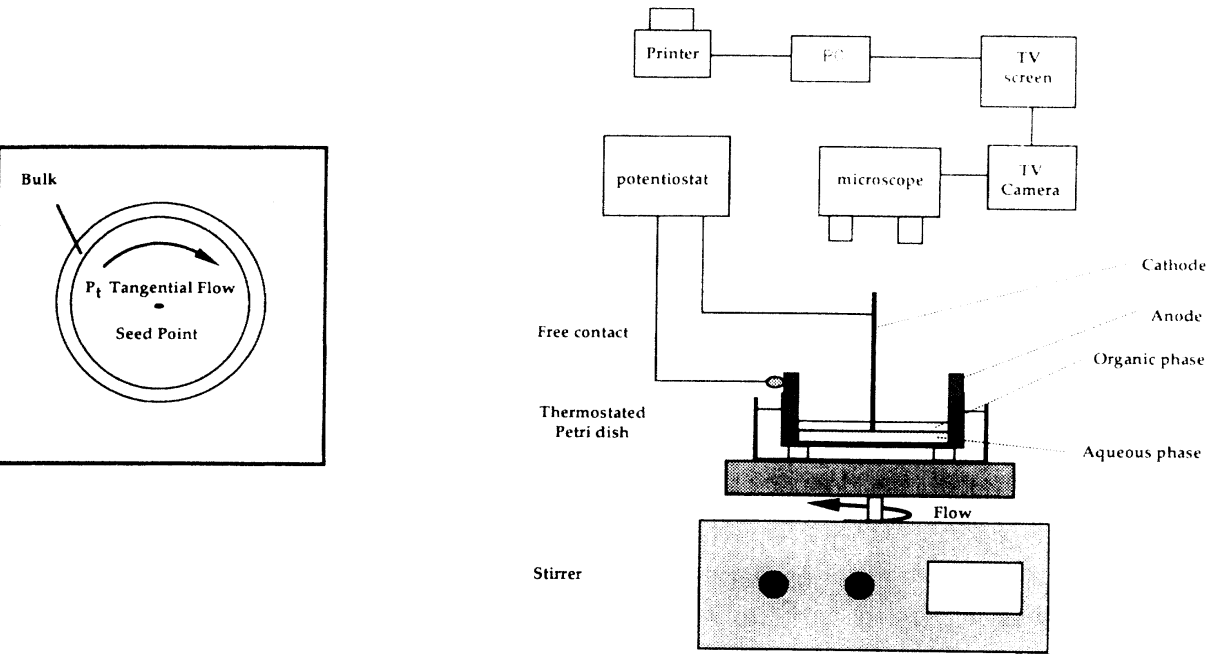

FIG. 1. (a) Scheme for the multiparticle DLA simulation under convective flow and (b) experimental setup (see text).

where $N$ is the total number of sites with radius $R$. $\rho_{R}(\theta)=1$ if there is a particle in the site defined by the radius $R$ and the angle $\theta$, and 0 otherwise. The summation in (2) is taken over $\theta^{\prime}$ values gradually increased by a fixed small $\Delta \theta^{\prime}$ from $\theta^{\prime}=0$ to $\theta^{\prime}=\pi$. According to (2), $C_{R}(\theta)$ describes the density-density correlation in a layer of width $\delta R$ (see caption of Fig. 4) at a distance $R$ from the origin as a function of the angle $\theta$, so that $\theta R$ is the distance separating two particles in the layer. These calculations were performed upon several radii and averaged over five different patterns.

\section{CONSTANT FLOW}

Figure 2 shows the effect of increasing tangential drift $P_{t}(0.0,0.2,0.6)$, while keeping constant the density parameter. In Figs. 2(a), 2(c), and 2(e), $C_{0}=0.05$ particles per site. As $P_{t}$ becomes higher, the overall shape of the cluster results in a less ramified structure and changes finally into a single arm, which is oppositely vortical to the tangential flow. This result is equivalent to the single needle observed in the strip geometry. ${ }^{12}$

In Figs. 2(b), 2(d), and 2(f), $C_{0}$ is maintained equal to 0.2 particles per site. As the tangential drift $P_{t}$ increases, the morphology of the cluster changes into a multiarm structure. The number of arms decreases, and they become more compact when $P_{t}$ increases. The arms are also oppositely vortical to the rotating flow.

We have also carried out a series of simulations keeping constant $P_{t}=0.6$ and varying $C_{0}$. As one could expect, the tendency of the cluster to change from a dense structure to a multiarm structure (and finally to a single arm) is observed.

\section{SHEAR FLOW}

Differences between constant and shear flows are shown in Fig. 3. All the simulations are made at $C_{0}=0.1$ particles per site. In Fig. 3(a) a pure multiparticle DLA pattern is shown for comparison. In Figs. 3(b) and 3(c), results from constant flow are represented. Figure 3(b) corresponds to a constant flow $=0.2$ and Fig. 3(c) to a constant flow $=0.6$. In Figs. $3(\mathrm{~d})$ and $3(\mathrm{e})$, the results of shear-flow simulations are shown. Figure $3(\mathrm{~d})$ presents results obtained with a shear flow of 0.2 and Fig. 3(e) with a shear flow of 0.6. The difference between constant and shear flows appears more prominent at high flow rates, where the constant flow develops single-arm patterns, while the shear flow still develops multiarm patterns.

\section{CORRELATION FUNCTION RESULTS}

Figure 4 shows the results of the tangential correlation function calculations for a pure multiparticle DLA pattern [Fig. 4(a)], a constant-flow pattern [Fig. 4(b)], and a shear-flow pattern [Fig. 4(c)] at a radius of 60 . The grown conditions are the following: maximum radius of patterns $=150, C_{0}=0.2$, and flow $=0.6$ for Figs. $4(\mathrm{~b})$ and 4(c). All three plots display both linear and oscillatory behaviors. For pure multiparticle DLA, the linear region extends over small angles, and the rest of the representation is dominated by the oscillatory region. For constant flux this linear region extends over a larger range of angles with an average monotonous decay, whereas for the shear flow we observe a mixed behavior between the first and second cases; that is, the linear region extends over a larger range of angles than in the pure multiparticle DLA, but the oscillatory region is more important than in the constant-flow plot. Our interpretation of these behaviors is as follows: The linear regions represent the values of the correlation function when sampling within one of the branches of the pattern, and the oscillatory region describes the correlation function extending to different branches of the pattern. As one could see in the figures presented above, patterns developed at high 


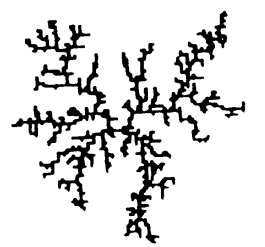

(a)

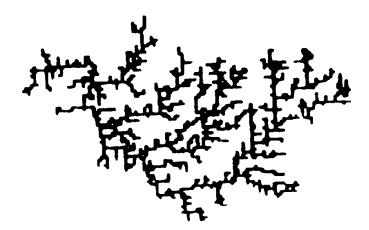

(c)
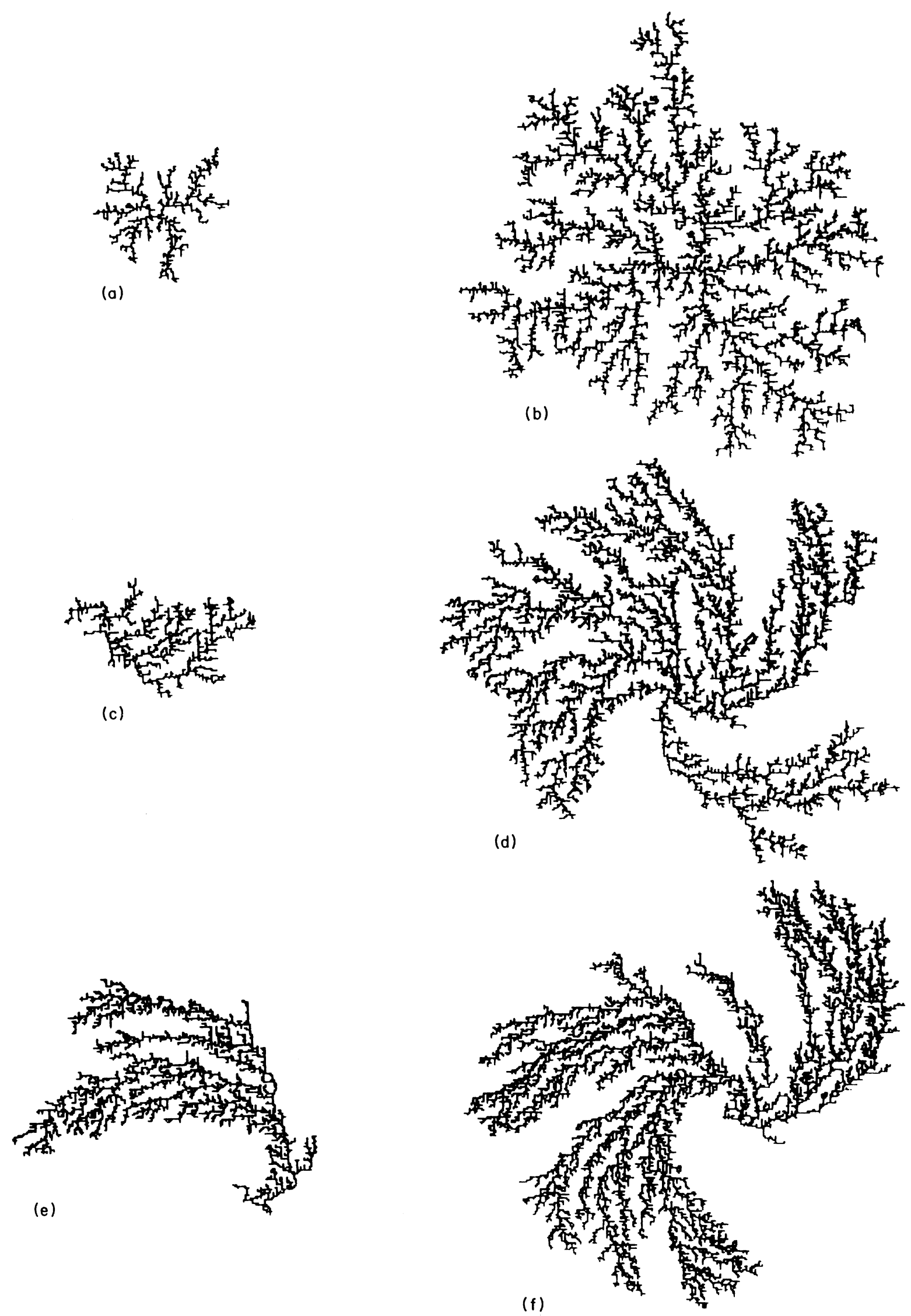

FIG. 2. Typical patterns grown by varying tangential-flow rates at two densities: (a),(c),(e) $C_{0}=0.05$ particles/site; (b),(d),(f) $C_{0}=0.2$ particles/site. Flow rates: (a),(b) 0.0 ; (c),(d) 0.2 ; (e),(f) 0.6 . 


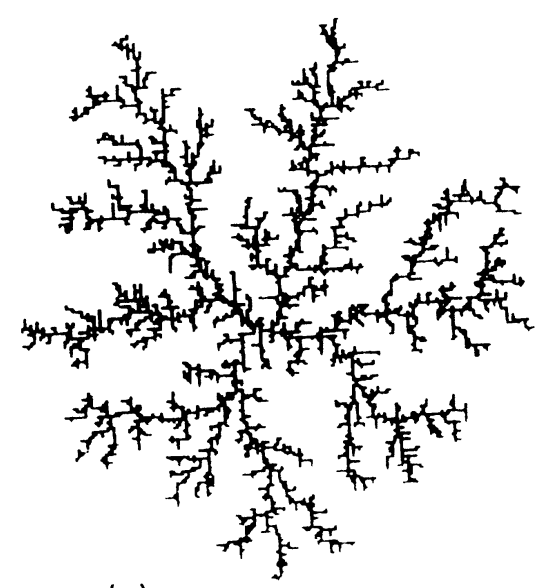

(a)

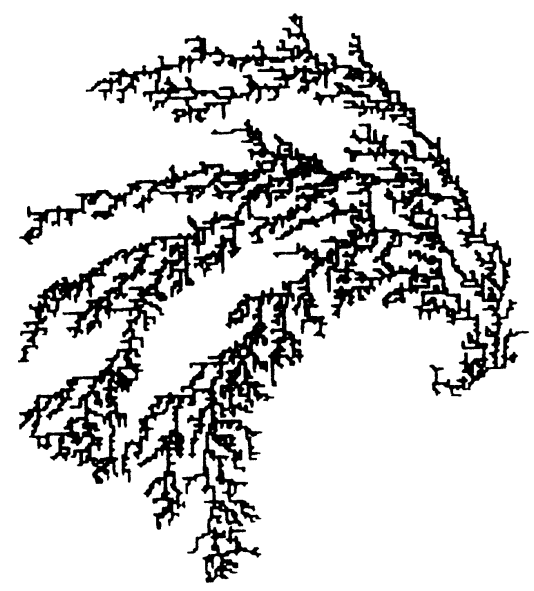

(c)
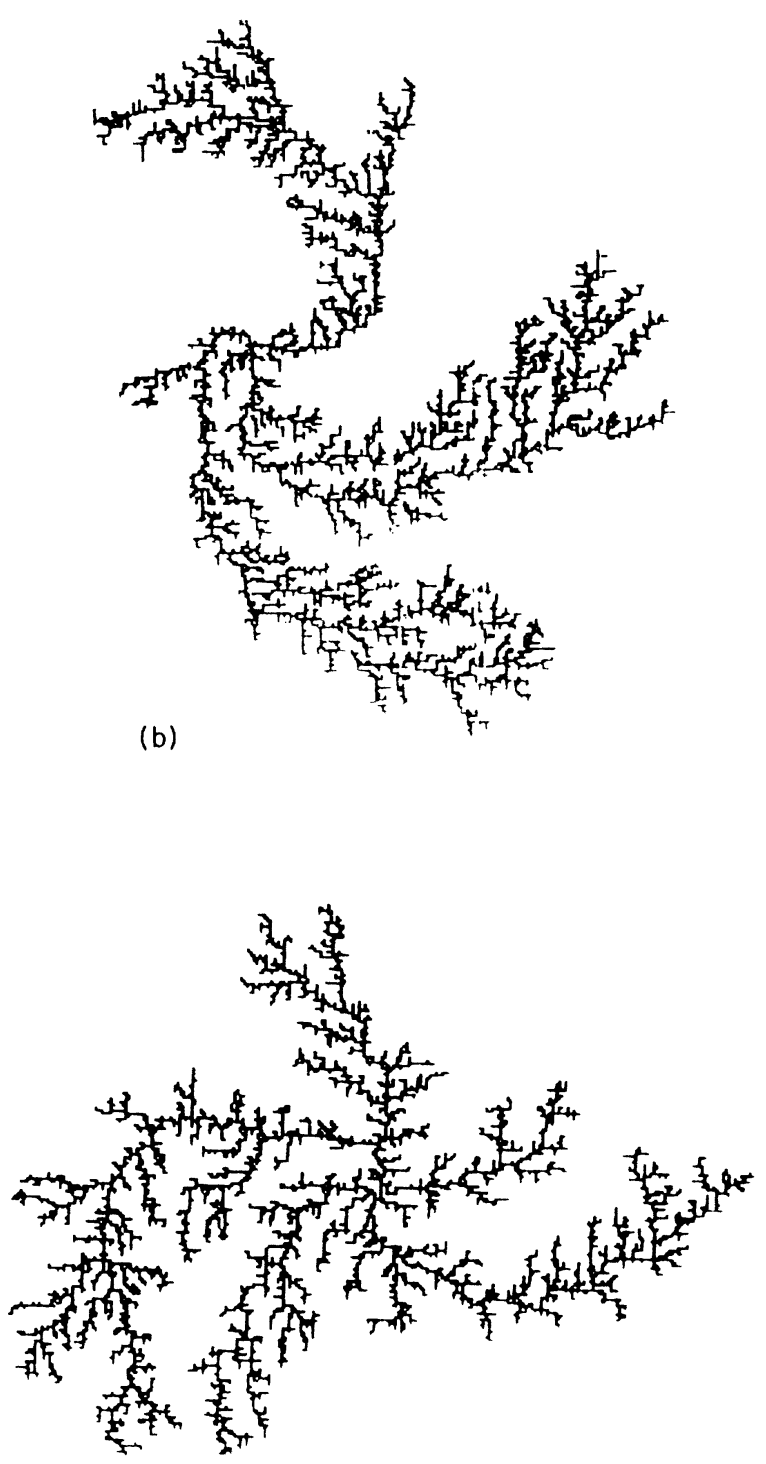

(d)

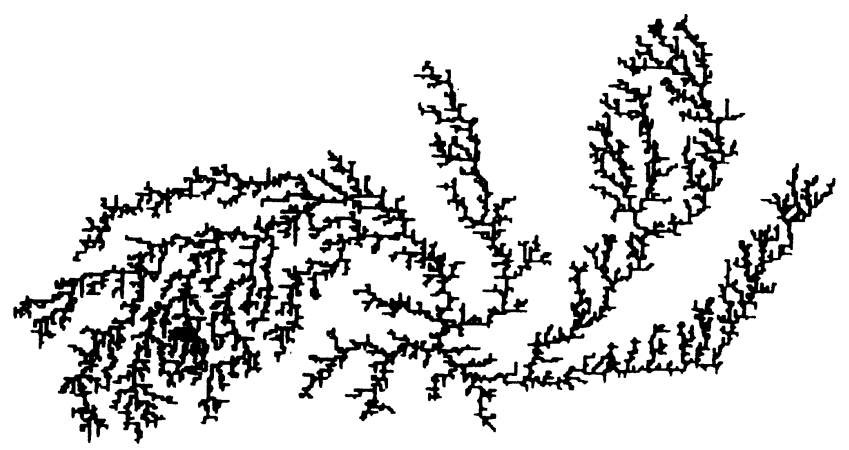

(e)

FIG. 3. Comparative figures of constant and shear flow. $C_{0}=0.1:$ (a) $P_{t}=0.0$; (b),(c) constant flow; (d),(e) shear flow. (b),(d) $P_{t}=0.2$; (c),(e) $P_{t}=0.6$. 
constant-flow rates tend to grow developing few arms patterns; that is, all particles are closely stuck onto single arms, and so the correlation function is linear for a larger range of angles. On the other hand, pure multiparticle DLA presents a multiarm structure, which explains the short range of angles where the linear behavior is observed and the extended oscillatory behavior at larger angles. As we have pointed out, shear-flow patterns have a multiarm structure under conditions where constant flow produces a single arm. The width of the shear-flow branches is larger than in the pure multiparticle DLA case [see Fig. 3(a)], and so we obtain a linear region that extends over a larger range of angles, but an oscillatory behavior is still present.

As a supplementary check, a finite-size scaling analysis for the pure DLA pattern shows that asymptotically the
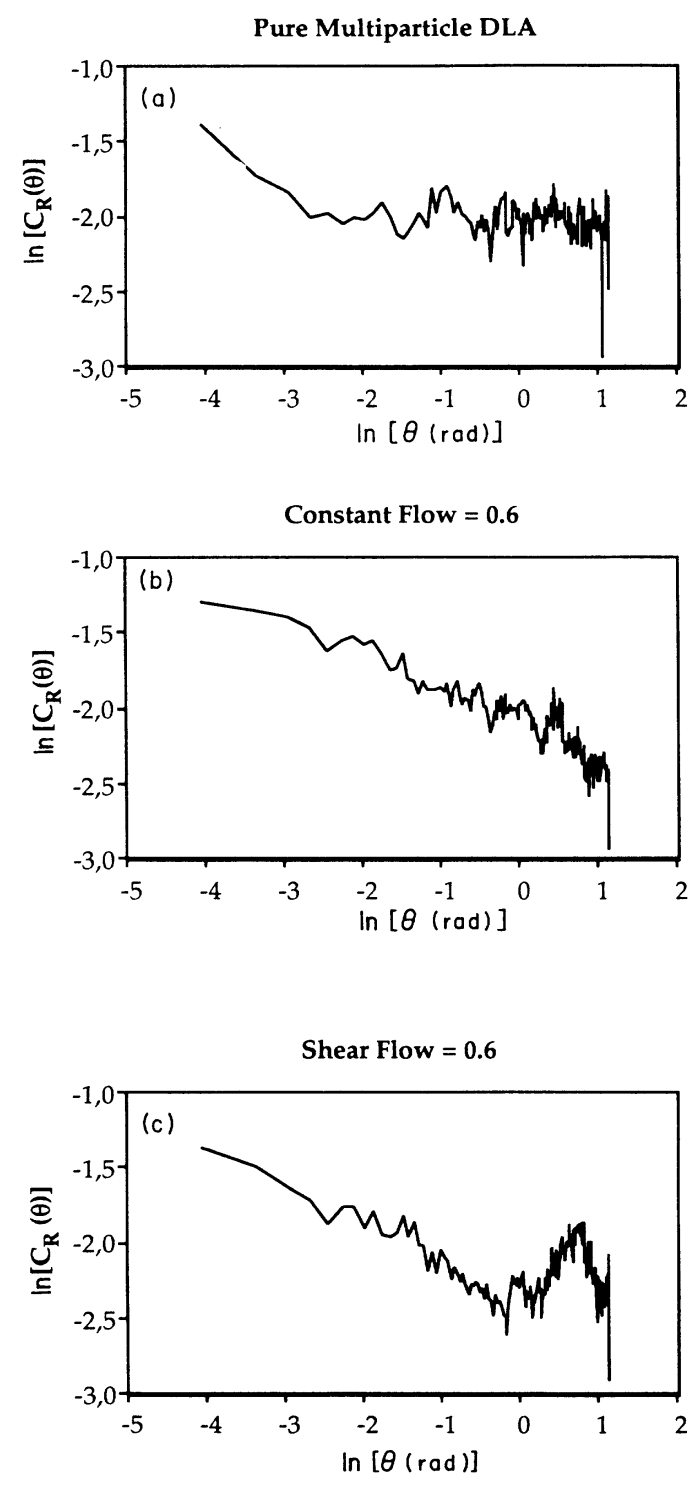

FIG. 4. Tangential correlations in multiparticle DLA. Clusters of approximately 10000 particles. The results were obtained in the interval $\delta R=R \pm \sqrt{2} / 2$ and averaging over five patterns. tangential correlation function scales as a function of $\theta$ with an exponent $\alpha_{1} \approx 0.43$, in agreement with that calculated by Vicsek. ${ }^{10}$

The use of the tangential correlation function analysis provides us with an important tool for the determination

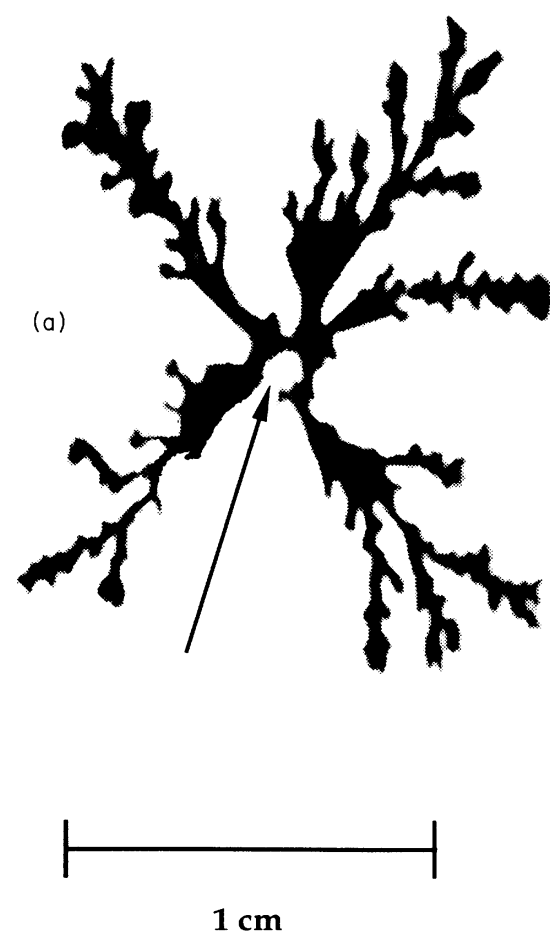

(b)

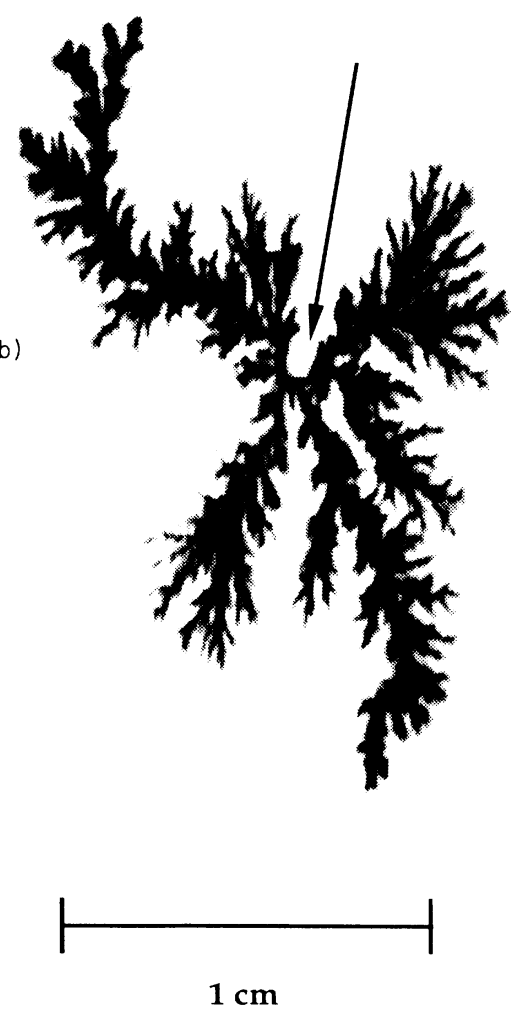

FIG. 5. Typical $\mathrm{Zn}$ electrodeposits. (a) is obtained without external convection and (b) with a superimposed flow (see text). The arrow shows the space occupied by the cathode. 
of anisotropy on aggregates; in particular, it can be useful to characterize experimental patterns grown under forced convection, as those we report on in what follows.

\section{EXPERIMENTAL RESULTS}

The experiments consisted of electrodepositing zinc on an interface formed by two inmiscible liquids, in our case $\mathrm{ZnSO}_{4}$ aqueous solution and buthyl acetate. The growth took place in a Petri dish $\sim 8 \mathrm{~cm}$ in diameter, provided with a thermostating jacket, which was placed on the plate of the stirrer [see Fig. 1(b)]. The cathode is a graphite bar of $0.5 \mathrm{~mm}$ in diameter, centered with respect to the cell. The anode consists of a $\mathrm{Zn}$ ring of $99.99 \%$ purity $\sim 8 \mathrm{~cm}$ in external diameter and $3 \mathrm{~mm}$ in width. Prior to each experiment, the graphite cathode was polished, and the $\mathrm{Zn}$ ring cleaned with a $\mathrm{HNO}_{3}$ solution. Analytical reagent-grade chemicals were used without further purification. Each experiment was performed according to the following procedure: $5 \mathrm{ml}$ of organic liquid was added gently onto $5 \mathrm{ml}$ of $\mathrm{ZnSO}_{4}$ aqueous solution in the cell, so that the surface of the aqueous phase was completely covered by the organic liquid. Immediately, the stirrer was set on and the plate would start to rotate at the prescribed velocity. After an applied cell voltage had been selected on a potentiosat, the tip of the cathode was carefully positioned exactly at the interface by using a micromanipulator.

Figure 5(a) shows a typical deposit obtained without external convection. When a superimposed flow is added, the obtained deposit is as shown in Fig. 5(b). The experimental conditions for both figures are $0.8 \mathrm{M} \mathrm{ZnSO}_{4}, 3.4$ $\mathrm{V}$, and $25^{\circ} \mathrm{C}$, and for the deposit obtained under convection, a radial velocity of $6 \mathrm{rpm}$ is chosen. One can observe in Fig. 5(b) the breaking of the radial symmetry of the pattern, whose arms tend to be spirals, in a similar way as what was predicted according to the simulation results reported above.

An exhaustive set of similar experiments, trying to test as much as possible the space of accessible parameters, concentration, voltage, and rotation rate, while improving the reproducibility of the observed patterns, is presently being conducted, and the results will be published elsewhere.

\section{ACKNOWLEDGMENTS}

We would thank Dr. Nagatani for useful discussions during his stay in Barcelona. We also thank P. P. Trigueros for helpful advice. Financial support from Dirección General de Investigacion Científica y Técnica (Spain) (project PB90-0455) is also acknowledged.
${ }^{1}$ T. A. Witten and L. M. Sander, Phys. Rev. Lett. 47, 1400 (1981); Phys. Rev. B 27, 5686 (1983).

${ }^{2}$ Kinetics of Aggregation and Gelation, edited by F. Family and D. P. Landau (North-Holland, Amsterdam, 1984).

${ }^{3}$ On Growth and Form, edited by H. E. Stanley and N. Ostrowsky (Nijhoff, The Hague, 1985).

${ }^{4}$ Fractals in Physics, edited by L. Pietronero and E. Tosatti (North-Holland, Amsterdam, 1986).

${ }^{5}$ H. J. Herrmann, Phys. Rep. 136, 153 (1986).

${ }^{6} \mathrm{P}$. Meakin, in Phase Transitions and Critical Phenomena, edited by C. Domb and J. L. Lebowitz (Academic, New York, 1988), Vol. 12 , p. 336.

${ }^{7}$ R. Julien and R. Botet, Aggregation and Fractal Aggregates (World Scientific, Singapore, 1987).

${ }^{8}$ J. Feder, Fractals (Plenum, New York, 1988).

${ }^{9}$ Random Fluctuations and Pattern Growth, edited by H. E. Stanley and N. Ostrowsky (Kluwer Academic, Dordrecht,
1988).

${ }^{10}$ T. Vicsek, Fractal Growth Phenomena (World Scientific, Singapore, 1989).

${ }^{11}$ P. Meakin, Phys. Rev. B 28, 5221 (1983).

${ }^{12}$ T. Nagatani and F. Sagués, Phys. Rev. A 43, 2970 (1991).

${ }^{13}$ R. F. Voss, Phys. Rev. B 30, 334 (1984).

${ }^{14}$ P. Meakin and J. M. Deutch, J. Chem. Phys. 80, 2115 (1984).

${ }^{15}$ R. F. Voss and M. Tomkievicz, J. Electrochem. Soc. 132, 371 (1985).

${ }^{16}$ T. Vicsek, Phys. Rev. A 32, 3084 (1985).

${ }^{17}$ L. P. Kadanoff, J. Stat. Phys. 39, 267 (1985).

${ }^{18}$ R. M. Brady and R. C. Ball, Nature 309, 225 (1984).

${ }^{19}$ Y. Sawada, A. Dougherty, and J. P. Gollub, Phys. Rev. Lett. 56, 1260 (1986).

${ }^{20}$ S. Miyazima (private communication).

${ }^{21}$ T. Nagatani and F. Sagués, J. Phys. Soc. Jpn. 59, 3447 (1990). 
(a) SIMULATION SCHEME

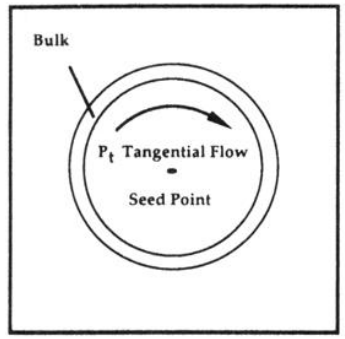

(b) EXPERIMENTAI SET UP

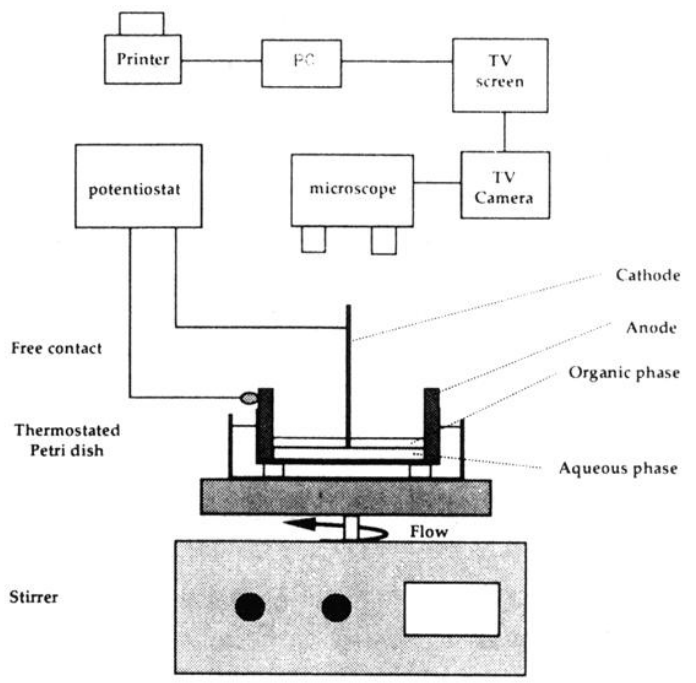

FIG. 1. (a) Scheme for the multiparticle DLA simulation under convective flow and (b) experimental setup (see text). 

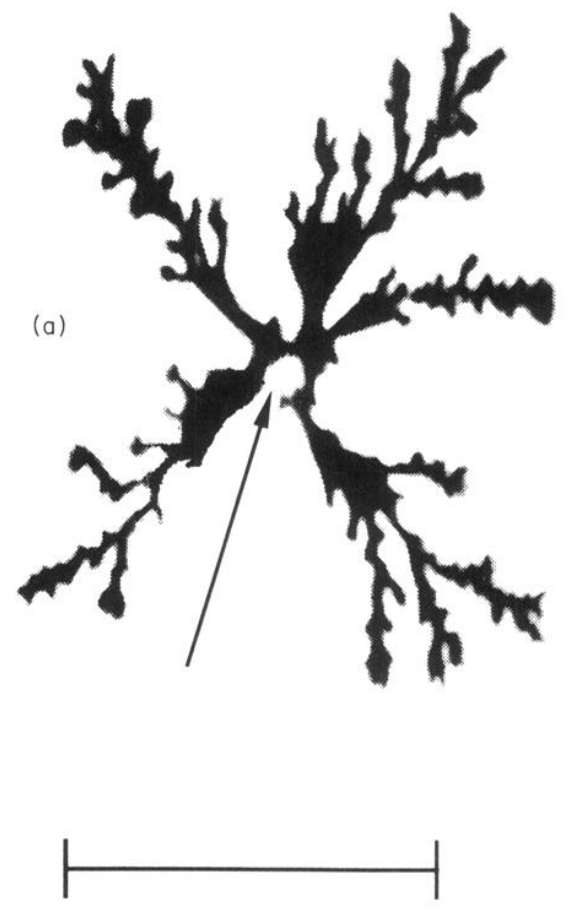

$1 \mathrm{~cm}$

(b)
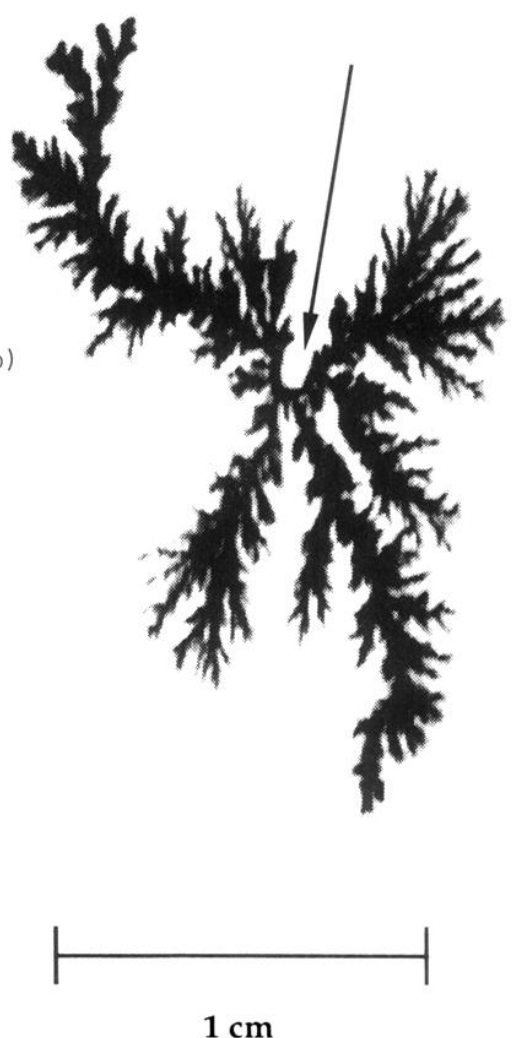

FIG. 5. Typical $\mathrm{Zn}$ electrodeposits. (a) is obtained without external convection and (b) with a superimposed flow (see text). The arrow shows the space occupied by the cathode. 